Textures and Microstructures, Vol. 34, pp. 63-64 Reprints available directly from the publisher Photocopying permitted by license only
(C) 2000 OPA (Overseas Publishers Association) N.V. Published by license under the Gordon and Breach Science Publishers imprint. Printed in Malaysia.

\title{
PREFACE
}

\section{TEXTURE IN FILMS AND COATINGS}

This symposium discusses advances in the area of texture control in coatings and films for the electronic, automobile, and magnetic recording industries and for other applications. Examples illustrating how the texture and structure of thin films and coatings are formed and how these can be controlled during manufacturing are discussed. Research on the processing of texture in coatings by various techniques is presented. Also included are presentations on various applications of texture control in coatings and films.

It is important to stress at this point that the research on texture of films is becoming increasingly important, the reason being that the texture engineering may optimize and improve properties of various materials used by electronic industry and to protect traditional engineering materials by the design of special textured coatings. One can mention here, as examples, textured $\mathrm{Al}-\mathrm{Cu}$ interconnects in electronic circuit boards, textured high $T_{c}$ superconductive films, textured $\mathrm{Zn}$ based automobile coatings and textured magnetic films for recording media.

When recognizing the role of texture in optimizing properties of films one has to admit that our understanding of processes of texture development in films is still in its infancy and an influence of substrate orientation, stress, temperature of film deposition and other parameters on texture development has to be better understood.

Texture development in films depends on competition between nuclei of different orientation and competition of grain growth. The rules of this competition have to be further studied and a knowledge of surface free energy, stress energy, energy of interfaces and mobility of atoms in 
films is of importance in such studies. Unfortunately, many of these parameters are still largely unknown because they are difficult to measure. Calculations that were performed to obtain some of these parameters using various theoretical methods are not yet sufficiently reliable.

There are many as yet unanswered questions related both to the understanding of texture development in films and correlation between texture and properties. The papers presented in this symposium illustrate both difficulties and progress in research on texture of films.

Jerzy A. Szpunar 